\title{
Extended Stochastic Transportation Problem with Flexible Inputs
}

\author{
${ }^{1}$ Gedam V.K. and ${ }^{2}$ Mahamad B. Pathan. \\ ${ }^{I}$ Department of Statistics, Savitribai Phule Pune University, Pune, Maharashtra, India. \\ ${ }^{2}$ Department of Statistics, Poona College of Arts, Science and Commerce, Pune, Maharashtra, India.
}

\begin{abstract}
The distribution of homogeneous goods in classical transportation problem is based on only per unit transportation cost. Usually, the decision of transporting homogeneous goods is not only based on transportation cost but also depends on many factors such as order size, total shipment-cost, profit, distance, man-power, etc. These factors are treated as inputs and outputs variables for each shipment link. Hence, the transportation problem with multiple inputs and outputs per shipment link is considered as Extended Transportation Problem. In the transportation problem, the distribution depends on the demand and the availability of homogeneous goods. If Demand and Availability of goods are not known to decision maker, the problem is known as Extended Stochastic Transportation Problem (ESTP). In this paper, we assume that the demand and availability are random variables with some known probability distribution. We proposed a method based on data envelopment analysis to decide optimal shipment plan for ESTP. The hypothetical illustration is considered to check the applicability of the proposed approach.
\end{abstract}

Keywords: Transportation problem; extended transportation problem; extended stochastic transportation problem; decision making units; data envelopment analysis; relative efficiency.

\section{Introduction}

The main purpose of transportation problem is to decide shipment plan of homogeneous commodity from various origins to various destinations such that the total transportation cost will be minimum. Only cost or profit is taken into account during the formulation of classical transportation problem. HitchcockF.L.[12] studied the classical transportation problem. Several kinds of variables such as cost, distance, time, shipment value, manpower, profit, order size, etc. per shipment link may be involved in transportation problem. These variables may be classified as a set of input or output variables. Such problem with multiple inputs and multiple outputs per shipment link is treated as Extended Transportation Problem (ETP). Each shipment link in the extended transportation problem is considered as Decision Making Unit (DMU). Chen L.H. and Lu H.W. [7] extended the assignment problem by considering multiple inputs and outputs. Alireza Amirtemoori[2] used the idea of Chen and $\mathrm{Lu}$ and suggested the method to solve extended transportation problem. The method proposed by Alireza Amirtemoori is based on CCR model, suggested by Charnes A, Cooper W.W, Rhodes E.[6 ]. CCR model is based on constant returns to scale (i.e increase in input variable causes increase in output variable and vice-versa). This assumption may not hold true in real life. So, Gedam V.K.and Pathan M.B. [8,9] have studied the extended transportation problem by using BCC model, suggested by Banker, Charnes, and Cooper [3], assume the variable returns to scale among input and output variables.

\section{Literature Review}

The Transportation problem with multi-choice cost per shipment link is named as 'Multi-Objective Problem'. Roy S.K. et al. $[14,15]$ have studied the multi-objective problem by assuming availability and demand stochastic in nature. Multi-objective problem involving general form of probability distributions studied by Abdul Quddoos et al. [1].Progressive review and analytical approach for optimal solution of stochastic transportation problems involving multi-choice cost was given by Maurya V.N. et al. [13]. It is observed that researchers took into account only multi-choice cost per shipment link where as we assume multiple inputs and multiple outputs variable per shipment link.

In the literature review, it is observed that Alireza Amirtemoori [2], Gedam V.K and Pathan M.B. [8, 9] have studied the extended transportation problem with known values for availability and demand per shipment link. In many real situations, the Decision Maker (DM) took decision about shipment without prior knowledge of availability and demand, which motivates us to study the problem when the demand and availability are unknown. We consider the availability and demand as random variables rather than the deterministic. These random variables are assumed to follow some known probability distribution. Under uncertainty of demand and availability, the problem is treated as Extended Stochastic Transportation Problem (ESTP).

Gedam V.K. and Pathan M.B. [10, 11] have suggested a method to solve ESTP using CCR model. They assumed the constant returns to scale relation among input and output variables. We proposed another approach for the distribution of homogeneous goods in ESTP on the basis of variable returns to scale relation 
among input and output variables. The optimal shipment plan is decided on the basis of efficiency of each DMUs. The efficiency of each DMU is calculated by using BCC model, suggested by Banker R.D., Charnes A. and Cooper W.W. [3], assume the variable returns to scale among input and output variables. The shipment plan with maximum efficiency is considered as an optimal plan to ESTP.

\section{Input Oriented BCC Model}

Consider $p$ as the number of DMUs under study. Suppose each DMU consumes varying amounts of $s$ different inputs to produce $t$-different outputs. Let $y_{r j} r=1,2, \ldots t$ and $x_{k j} ; k=1,2, \ldots . . s$ denotes the non-negative input and output values respectively for $h^{\text {th }}$ DMU denoted as $\mathrm{DMU}_{h} \quad ; \quad h=1,2, \ldots .$. . One of the DMUs is considered for evaluation, denoted as $\mathrm{DMU}_{\mathrm{o}}$, and placed in the functional form to maximize output, while also leaving it in the constraints. We have input oriented BCC model as follows.

Model 1:

$$
\text { Maximise } \boldsymbol{e}_{o}=\sum_{r=1}^{t} \boldsymbol{u}_{r} \boldsymbol{y}_{r o}
$$

Subject to:

$$
\begin{aligned}
& \mathcal{V}_{*}+\sum_{k=1}^{s} \mathcal{V}_{k} \boldsymbol{X}_{k o}=1 \\
& \sum_{r=1}^{t} \boldsymbol{u}_{r} \boldsymbol{y}_{r h}-\boldsymbol{v}_{*}-\sum_{k=1}^{s} \boldsymbol{v}_{k} \boldsymbol{x}_{k h} \leq 0 \quad ; h=1,2, \ldots, p \\
& u_{r} \geq 0 \quad ; r=1,2, \ldots t \quad, v_{k} \geq 0 \quad ; k=1,2, \ldots S \\
& V_{*} \text { is unrestricted. }
\end{aligned}
$$

\section{Extended Stochastic Transportation Problem}

Consider the transportation problem with $m$-warehouses and $n$-destinations. Let $i^{\text {th }}$ warehouse has availability of $A_{i}$ units of homogeneous commodity. Let $j^{\text {th }}$ destination has requirements of $B_{j}$ units of homogeneous commodity. The availability $A_{i}, i=1,2, \ldots m$ and demand $B_{j}, j=1,2, \ldots n$ are random variables with some known probability distribution. Let $\mathcal{X}_{i j}^{(k)} ; k=1,2,3, \ldots s$ denotes $s$-inputs and $y_{i j}^{(r)}$;

\begin{tabular}{|c|c|c|c|c|c|}
\hline \multirow{2}{*}{$\begin{array}{c}\text { Origins/ } \\
\text { Warehouses }\end{array}$} & \multicolumn{4}{|c|}{ Destinations } & \multirow{2}{*}{ Availability } \\
\hline & $D_{1}$ & $D_{2}$ & ............ & $D_{n}$ & \\
\hline$O_{l}$ & $\left(x_{11}^{(k)}, y_{11}^{(r)}\right)$ & $\left(x_{12}^{(k)}, y_{12}^{(r)}\right)$ & ……...... & $\left(x_{1 n}^{(k)}, y_{1 n}^{(r)}\right)$ & $A_{1}$ \\
\hline $\mathrm{O}_{2}$ & $\left(x_{21}^{(k)}, y_{21}^{(r)}\right)$ & $\left(x_{22}^{(k)}, y_{22}^{(r)}\right)$ & ............. & $\left(x_{2 n}^{(k)}, y_{2 n}^{(r)}\right)$ & $A_{2}$ \\
\hline . & .. & $\ldots$ & ........... & $\ldots$ & \\
\hline$O_{m}$ & $\left(x_{m 1}^{(k)}, y_{m 1}^{(r)}\right)$ & $\left(x_{m 2}^{(k)}, y_{m 2}^{(r)}\right)$ & .......... & $\left(x_{m n}^{(k)}, y_{m n}^{(r)}\right)$ & $A_{m}$ \\
\hline Demand & $B_{1}$ & $B_{2}$ & ........... & $B_{n}$ & \\
\hline
\end{tabular}
$r=1,2,3 \ldots t$ denotes $t$-outputs for $(i, j)^{t h}$ shipment link respectively. It is assumed that the inputs and outputs for each link are known to decision maker. Such problem is known as 'Extended Stochastic Transportation Problem (ESTP)'. We represent ESTP in Table 1.

Table 1: Extended Stochastic Transportation Problem 


\section{Mathematical Formulation of ESTP}

Let $\boldsymbol{t}_{i j}$ represents the number of units shipped from $i^{\text {th }}$ warehouse to $j^{\text {th }}$ destination. Let $e_{i j}$ be the efficiency of shipment link (DMU) from $i^{\text {th }}$ warehouse to $j^{\text {th }}$ destination. Under this assumption, we write the model for ESTP given in Table1 as,

Model 2:

Minimise $\sum_{i=1}^{m} \sum_{j=1}^{n}\left(1-e_{i j}\right) t_{i j}$

Subject to:

$$
\begin{array}{cl}
\operatorname{Pr}\left[\sum_{j=1}^{n} t_{i j} \leq A_{i}\right] \geq 1-\alpha_{i} & ; i=1,2, \ldots \ldots . m \\
\operatorname{Pr}\left[\sum_{i=1}^{m} t_{i j} \geq B_{j}\right] \geq 1-\beta_{j} \quad ; j=1,2, \ldots \ldots . . n \\
t_{i j} \geq 0
\end{array}
$$

Where,

$0<\alpha_{i}<1, \forall_{i}$ and $0<\beta_{j}<1, \forall_{j}$ are the specified probability levels.

The optimal shipment plan is decided by solving model 2 with following three cases.

Case 1: Assuming only availability $A_{i} ; i=1,2, \ldots m$ follows normal distribution.

Case 2: Assuming only demand $\boldsymbol{B}_{j} ; j=1,2, \ldots n$ follows normal distribution.

Case3: Assuming both availability $A_{i} ; i=1,2, \ldots m$ and demand $B_{j} ; j=1,2, \ldots n$ follows normal distributions.

So consider,

\section{Case 1: Only availability follows normal distribution}

The constraints (7) in model 2 can be represented as below.

$$
\operatorname{Pr}\left[A_{i} \leq \sum_{j=1}^{n} t_{i j} \leq \alpha_{i}, \quad i=1,2, \ldots \ldots . m .\right.
$$

It is assumed that $A_{i} ; i=1,2, \ldots m$ follows normal distribution with parameters mean as $\mu_{i}, i=1,2, \ldots, m$ and variances as $\sigma_{i}^{2}, i=1,2, \ldots, m$. So we have probability density function of $A_{i} ; i=1,2, \ldots m$ as below:

$$
f\left(a_{i}, \mu_{i}, \sigma_{i}^{2}\right)=\frac{1}{\sigma_{i} \sqrt{2 \pi}} e^{-\frac{1}{2}\left(\frac{a_{i}-\mu_{i}}{\sigma_{i}}\right)^{2}} ; a_{i} \in R, \mu_{i} \in R, \sigma_{i}>0
$$

Now the equation (10) can be written as

$$
\begin{aligned}
& \int_{-\infty}^{\sum_{j=1}^{n} t_{i j}} \frac{1}{\sigma_{i} \sqrt{2 \pi}} \exp \left\{-\frac{1}{2}\left(\frac{a_{i-\mu_{i}}}{\sigma_{i}}\right)^{2} d\left(a_{i}\right) \leq \alpha_{i}\right. \\
& \text { Put } Z_{i}=\left(\frac{a_{i}-\mu_{i}}{\sigma_{i}}\right), \text { we write above integral as }
\end{aligned}
$$




$$
\begin{aligned}
& \left(\frac{\sum_{j=1}^{n} t_{i j}-\mu_{i}}{\sigma_{i}}\right) \\
& \int_{-\infty} \frac{1}{\sqrt{2 \pi}} \exp \left\{-\frac{1}{2} z_{i}^{2}\right\} d\left(z_{i}\right) \leq \alpha_{i} \\
& \Phi\left(\frac{\sum_{j=1}^{n} t_{i j}-\mu_{i}}{\sigma_{i}}\right)-\Phi(-\infty) \leq \alpha_{i} \\
& \sum_{j=1}^{n} t_{i j} \leq \mu_{i}+\sigma_{i} \Phi^{-1}\left(\alpha_{i}\right), \quad i=1,2, \ldots, m .
\end{aligned}
$$

So, we have obtained the deterministic transportation problem (model 3) instead of ESTP (model 2) as follows: Model 3:

$$
\text { Minimise } \sum_{i=1}^{m} \sum_{j=1}^{n}\left(1-e_{i j}\right) t_{i j}
$$

Subject to:

$$
\begin{array}{ll}
\sum_{j=1}^{n} \boldsymbol{t}_{i j} \leq \boldsymbol{\mu}_{i}+\sigma_{i} \Phi^{-1}\left(\alpha_{i}\right), & i=1,2, \ldots, m \\
\sum_{i=1}^{m} \boldsymbol{t}_{i j} \geq \boldsymbol{b}_{j}, & j=1,2, \ldots, n \\
\boldsymbol{t}_{i j} \geq 0, & \forall i \text { and } j .
\end{array}
$$

\section{Case 2: Only demand follows normal distribution}

The constraints (8) in model 2 can be represented as below.

$$
\operatorname{Pr}\left[\sum_{i=1}^{m} t_{i j} \leq B_{j}\right] \leq \beta_{j}, \quad j=1,2, \ldots n
$$

It is assumed that $\boldsymbol{B}_{j} ; j=1,2, \ldots n$ follows normal distribution with parameters means as $\mu_{j}^{\prime}, j=1,2, \ldots, n$ and variances as $\sigma_{j}^{\prime 2}, j=1,2, \ldots, n$. So we have probability density function of $\boldsymbol{B}_{j} ; j=1,2, \ldots n$ as below:

$$
f\left(b_{j}, \mu_{j}^{\prime}, \sigma_{j}^{\prime 2}\right)=\frac{1}{\sigma_{j}^{\prime} \sqrt{2 \pi}} e^{-\frac{1}{2}\left(\frac{b_{j}-\mu_{j}^{\prime}}{\sigma_{j}^{\prime}}\right)^{2}} ; b_{j} \in R, \mu_{j}^{\prime} \in R, \sigma_{j}^{\prime}>0
$$

Now the equation (20) can be written as

$$
\int_{i=1}^{\infty} \frac{1}{\sigma_{i j}^{\prime} t_{j} \sqrt{2 \pi}} \exp \left\{-\frac{1}{2}\left(\frac{b_{j}-\mu^{\prime}{ }_{j}}{\sigma^{\prime}{ }_{j}}\right)^{2}\right\} d\left(b_{j}\right) \leq \beta_{j}
$$


Put $z_{j}=\left(\frac{b_{j-\mu^{\prime}}}{\sigma^{\prime}{ }_{j}}\right)$, we write above integral as

$\left(\begin{array}{l}\sum_{i=1}^{m} t_{i j}{ }^{\prime} \mu_{j}^{\prime} \\ \sigma^{\prime}{ }_{j}\end{array}\right)^{\infty} \frac{1}{\sqrt{2 \pi}} \exp \left\{-\frac{1}{2} z_{j}^{2}\right\} d\left(z_{j}\right) \leq \beta_{j}$

$\Phi(\infty)-\Phi\left(\frac{\sum_{i=1}^{m} t_{i j}-\mu_{j}^{\prime}}{\sigma_{j}^{\prime}}\right) \leq \beta_{j}$

$\sum_{i=1}^{m} t_{i j} \geq \mu_{j}^{\prime}+\sigma_{j}^{\prime} \Phi^{-1}\left(1-\beta_{j}\right)$

So, we have obtained the deterministic transportation problem (model 4) instead of ESTP (model 2) as follows: Model 4:

$$
\text { Minimise } \sum_{i=1}^{m} \sum_{j=1}^{n}\left(1-e_{i j}\right) t_{i j}
$$

Subject to:

$$
\begin{array}{ll}
\sum_{j=1}^{n} t_{i j} \leq a_{i}, & i=1,2, \ldots, m \\
\sum_{i=1}^{m} t_{i j} \geq \mu_{j}^{\prime}+\sigma_{j}^{\prime} \Phi^{-1}\left(1-\beta_{j}\right), & j=1,2, \ldots, n \\
t_{i j} \geq 0, & \forall i \text { and } j .
\end{array}
$$

Case 3: Both availability and demand follows normal distribution

Suppose availability $A_{i} ; i=1,2, \ldots m$ follows normal distribution with parameters means as $\mu_{i}, i=1,2, \ldots, m$ and variances as $\sigma_{i}^{2}, i=1,2, \ldots, m$. The probabilistic constraints (7) of model 2 are converted to deterministic constraints (15).

Similarly, demand $\boldsymbol{B}_{j} ; j=1,2, \ldots n$ follows normal distribution with parameters means as $\mu^{\prime}{ }_{j}, j=1,2, \ldots, n$ and variances as $\sigma_{j}^{\prime 2}, j=1,2, \ldots, n$. The probabilistic constraints (8) of model 2 are converted to deterministic constraints (25).

Hence, we write deterministic transportation problem (model 5) instead of ESTP (model 2) as follows.

Model 5:

$$
\text { Minimise } \sum_{i=1}^{m} \sum_{j=1}^{n}\left(1-e_{i j}\right) t_{i j}
$$

Subject to:

$$
\sum_{j=1}^{n} t_{i j} \leq \mu_{i}+\sigma_{i} \Phi^{-1}\left(\alpha_{i}\right), \quad \quad i=1,2, \ldots, m
$$




$$
\begin{array}{ll}
\sum_{i=1}^{m} t_{i j} \geq \mu_{j}^{\prime}+\sigma_{j}^{\prime} \Phi^{-1}\left(1-\beta_{j}\right), & j=1,2, \ldots, n \\
t_{i j} \geq 0, & \forall i \text { and } j .
\end{array}
$$

$\Phi^{-1}($.$) is an inverse of cumulative distribution function of standard normal random variable.$

\section{Proposed method to solve ESTP}

We solve ESTP in two stages. The efficiencies for various shipment links (DMUs) are obtained in stage I and the optimal shipment plan with maximum efficiency is decided in stage II as discussed below.

\subsection{Efficiencies of various DMUs based on input oriented BCC model}

Consider each possible shipment link $(i, j)$ as a DMU in ESTP. For each warehouse $i$, we consider all destinations $j(j=1,2, . . n)$. With the warehouse $i$ as a target, the efficiency of the $(i, j)^{\text {th }}$ shipment link denoted as $\mathrm{DMU}^{(1)}$ can be determined by using the DEA technique (BCC model 1) as cited in section 3 . We have relative efficiency of $i^{\text {th }}$ warehouse:

$$
\boldsymbol{e}_{i j}^{(1)}=\text { Maximise }\left\{\sum_{r=1}^{t} \boldsymbol{u}_{r} y_{i j}^{(r)}\right\}
$$

Subject to:

$$
\begin{array}{cc}
\boldsymbol{v}_{*}+\sum_{k=1}^{s} \boldsymbol{v}_{k} \boldsymbol{x}_{i j}^{(k)}=1 & \\
\sum_{r=1}^{t} \boldsymbol{u}_{r} y_{i j}^{(r)}-\boldsymbol{v}_{*}-\sum_{k=1}^{s} \boldsymbol{v}_{k} \boldsymbol{X}_{i j}^{(k)} \leq 0 & ; j=1,2, \ldots n \\
\boldsymbol{u}_{r} \geq \boldsymbol{\varepsilon} & ; r=1,2, \ldots . t \\
\boldsymbol{v}_{k} \geq \boldsymbol{\varepsilon} & ; k=1,2, \ldots . . s \\
\boldsymbol{\varepsilon}>0 . &
\end{array}
$$

Using set of equations (34-39), we obtain the relative efficiency of $i^{\text {th }}$ warehouse as $e_{i 1}^{(1)}, e_{i 2}^{(1)}, \ldots . ., e_{i n}^{(1)} \quad$ by changing the target warehouse in the above set of equations.

Similarly, we have relative efficiency of $j^{\text {th }}$ destination with link $(i, j)$ denoted as $\operatorname{DMU}_{\mathrm{s}}^{(2)}$ :

$$
\boldsymbol{e}_{i j}^{(2)}=\operatorname{Maximise}\left\{\sum_{r=1}^{t} u_{r} y_{i j}^{(r)}\right\}
$$

Subject to:

$$
\mathcal{V}_{*}+\sum_{k=1}^{s} \mathcal{V}_{k} \boldsymbol{X}_{i j}^{(k)}=1
$$

$$
\begin{array}{cc}
\sum_{r=1}^{t} \boldsymbol{u}_{r} \boldsymbol{y}_{i j}^{(r)}-\boldsymbol{v}_{*}-\sum_{k=1}^{s} \boldsymbol{v}_{k} \boldsymbol{x}_{i j}^{(k)} \leq 0 & ; i=1,2, \ldots . m \\
u_{r} \geq \varepsilon & ; r=1,2, \ldots . t \\
v_{k} \geq \varepsilon & ; k=1,2, \ldots . s \\
\varepsilon>0 &
\end{array}
$$

Using set of equations (40-45), we obtain the relative efficiency of $j^{\text {th }}$ destination as $e_{1 j}^{(2)}, e_{2 j}^{(2)}, \ldots, e_{m j}^{(2)}$ by changing the target destination in the above set of equations.

We have determined the relative efficiency of all warehouses to each destination based on the set of decision making units $\mathrm{DMU}_{\mathrm{s}}^{(1)}$ and vice-versa for $\mathrm{DMU}_{\mathrm{s}}^{(2)}$. However, the two groups of relative efficiencies are obtained for the comparisons from either the warehouse side or the destinations side. For the transportation problem with multiple inputs and outputs, we need to optimize the total efficiency for entire shipment. We therefore construct a composite efficiency index to incorporate the two kinds of relative efficiencies. Hence, we suggest two ways to combine these two types of efficiency as below. 


$$
\boldsymbol{e}_{i j}^{*}=\frac{\boldsymbol{e}_{i j}^{(1)}+\boldsymbol{e}_{i j}^{(2)}}{2} ; i=1,2, \ldots m ; j=1,2, \ldots, n
$$

We consider $\boldsymbol{e}_{i j}^{*}$ as the composite efficiency index which represents the performance measure of each shipment link $(i, j)$.

Also, we define the performance measure of each shipment link $(i, j)$ by considering most efficient DMUs as below.

$$
\boldsymbol{e}_{i j}^{* *}=\operatorname{Maximum}\left\{\boldsymbol{e}_{i j}^{(1)}, \boldsymbol{e}_{i j}^{(2)}\right\} ; \text { for each shipment link }(i, j) .
$$

\subsection{Optimal solution to ESTP}

The ESTP (model 2) given in section 5 is converted to deterministic transportation problem by taking three possible cases as mentioned in section 5 .

In case 1, the availabilities are assumed to follow normal distribution where as demands are assumed to be known to DM. The model obtained under case 1(model 3) is a deterministic transportation problem.

In case 2, the availabilities are assumed to be known to DM where as demand follows normal distribution. The model obtained under case 2(model 4) is a deterministic transportation problem.

In case 3, both availability and demand are assumed to be independent random variables and follows normal distribution. The model obtained in this case (model 5) is also deterministic transportation problem.

The various models derived in section 5 are classical transportation problems and can be solved by using simplex algorithm. The models given in section 5 are solved to get an optimal shipment plan by replacing $\boldsymbol{e}_{i j}$ by $\boldsymbol{e}_{i j}^{*}$ respectively. Similarly, by putting another measure of performance $\boldsymbol{e}_{i j}^{* *}$ in place of $\boldsymbol{e}_{i j}$.

\section{Hypothetical Example}

Suppose a company produces two wheeler motor-bikes and sends the production to three warehouses. The production is distributed among four dealers. Each warehouse represents a potential point of supply and dealer as point of destination. Cost per unit of transportation is taken as input variables where as the shipment value and profit per unit are considered as output variable. Suppose these variables are known in advance and are given in Table 2. Each triplet in Table 2 indicates cost per unit of transportation, shipment value and profit per unit respectively.

Table 2: Input and Output values per shipment link

\begin{tabular}{|c|c|c|c|c|}
\hline \multirow{2}{*}{ Warehouse } & \multicolumn{4}{|c|}{ Dealers } \\
\cline { 2 - 5 } & $\mathrm{D}_{1}$ & $\mathrm{D}_{2}$ & $\mathrm{D}_{3}$ & $\mathrm{D}_{4}$ \\
\hline $\mathrm{W}_{1}$ & $(23,158,252)$ & $(25,198,267)$ & $(17,300,274)$ & $(15,255,354)$ \\
\hline $\mathrm{W}_{2}$ & $(25,205,227)$ & $(23,279,365)$ & $(23,271,399)$ & $(21,178,210)$ \\
\hline $\mathrm{W}_{3}$ & $(21,271,297)$ & $(25,245,390)$ & $(18,251,241)$ & $(24,221,377)$ \\
\hline
\end{tabular}

\subsection{Computation of Efficiencies}

The set of equations (34-39) given in section 6.1 are solved by using data given in Table 2. The calculations of efficiencies for set of DMUs ${ }^{(1)}$ are performed by row-wise. The various values of weights for input and output variables with efficiencies for the set of DMUs ${ }^{(1)}$ are given in Table 3.

Table 3: Efficiencies for set of DMUs ${ }^{(1)}$ with input and output weights

\begin{tabular}{|c|c|c|c|c|c|}
\hline DMUs $^{(1)}$ & $\boldsymbol{e}_{i j}^{(1)}$ & $\boldsymbol{u}_{1}$ & $\boldsymbol{u}_{2}$ & $\boldsymbol{v}_{*}$ & $\boldsymbol{v}_{1}$ \\
\hline$(1,1)$ & .7089 & .0001 & .0028 & .9977 & .0001 \\
\hline$(1,2)$ & .7659 & .0022 & .0012 & .9975 & .0001 \\
\hline$(1,3)$ & 1 & .0031 & .0003 & 0 & .0588 \\
\hline$(1,4)$ & 1 & .0035 & .0003 & 0 & .0667 \\
\hline$(2,1)$ & .7305 & .0035 & .0001 & .9975 & .0001 \\
\hline$(2,2)$ & 1 & .0027 & .0006 & 0 & .0435 \\
\hline$(2,3)$ & 1 & .0027 & .0006 & 0 & .0435 \\
\hline$(2,4)$ & 1 & .0055 & .0001 & -4.9955 & .2855 \\
\hline$(3,1)$ & 1 & .0011 & .0024 & 0 & .0476 \\
\hline$(3,2)$ & 1 & .0009 & .0019 & 0 & .04 \\
\hline$(3,3)$ & 1 & .0017 & .0024 & 0 & .0555 \\
\hline$(3,4)$ & 1 & .0004 & .0024 & 0 & .0416 \\
\hline
\end{tabular}


Similarly, the set of equations given in (40-45) are solved by using data given in Table 2 . The calculations of efficiencies for set of DMUs ${ }^{(2)}$ are performed by column-wise. The various values of weights for input and output variables with efficiencies for the set of DMUs ${ }^{(2)}$ are given in Table 4.

Table 4: Efficiencies for set of DMUs ${ }^{(2)}$ with input and output weights

\begin{tabular}{|c|c|c|c|c|c|}
\hline DMUs $^{(2)}$ & $\boldsymbol{e}_{i j}^{(2)}$ & $\boldsymbol{U}_{1}$ & $\boldsymbol{u}_{2}$ & $\boldsymbol{V}_{*}$ & $\boldsymbol{V}_{1}$ \\
\hline$(1,1)$ & .041 & .0001 & .0001 & 0 & .0435 \\
\hline$(2,1)$ & .0432 & .0001 & .0001 & 0 & .04 \\
\hline$(3,1)$ & .0568 & .0001 & .0001 & 0 & .0476 \\
\hline$(1,2)$ & .0465 & .0001 & .0001 & 0 & .04 \\
\hline$(2,2)$ & .0644 & .0001 & .0001 & 0 & .0435 \\
\hline$(3,2)$ & .0635 & .0001 & .0001 & 0 & .04 \\
\hline$(1,3)$ & .0574 & .0001 & .0001 & 0 & .0588 \\
\hline$(2,3)$ & .067 & .0001 & .0001 & 0 & .0435 \\
\hline$(3,3)$ & .0492 & .0001 & .0001 & 0 & .0555 \\
\hline$(1,4)$ & .0609 & .0001 & .0001 & 0 & .0666 \\
\hline$(2,4)$ & .0388 & .0001 & .0001 & 0 & .0476 \\
\hline$(3,4)$ & .0598 & .0001 & .0001 & 0 & .0417 \\
\hline
\end{tabular}

We put row-wise and also column-wise efficiency calculations in one table. In each cell of Table 5, first element indicates efficiency of DMUs ${ }^{(1)}$ and second element indicates efficiency of DMUs ${ }^{(2)}$. The performance of each decision making point (shipment link) is measure by converting two efficiency into a single efficiency as composite efficiency $\left(\boldsymbol{e}_{i j}^{*}\right)$. The composite efficiency $\boldsymbol{e}_{i j}^{*}$ for each shipment link $(i, j)$ is obtained by using equation (46) and tabulated in Table 6 .

Similarly, the most efficient DMU is decided by equation (47) denoted as $\boldsymbol{e}_{i j}^{* *}$ and are tabulated in Table 7.

Table 5: Efficiencies for both DMUs together

\begin{tabular}{|c|c|c|c|c|}
\hline \multirow{2}{*}{ Warehouse } & \multicolumn{4}{|c|}{ Dealers } \\
\cline { 2 - 5 } & $\mathrm{D}_{1}$ & $\mathrm{D}_{2}$ & $\mathrm{D}_{3}$ & $\mathrm{D}_{4}$ \\
\hline \multirow{2}{*}{$\mathrm{W}_{1}$} & .7089 & .7659 & 1 & 1 \\
\cline { 2 - 5 } & .041 & .0465 & .0574 & .0609 \\
\hline \multirow{2}{*}{$\mathrm{W}_{2}$} & .7305 & 1 & 1 & 1 \\
\cline { 2 - 5 } & .0432 & .0644 & .067 & .0388 \\
\hline \multirow{2}{*}{$\mathrm{W}_{3}$} & 1 & 1 & 1 & 1 \\
\cline { 2 - 5 } & .0568 & .0635 & .0492 & .0598 \\
\hline
\end{tabular}

Table 6: Composite efficiency index $\left(\boldsymbol{e}_{i j}^{*}\right)$

\begin{tabular}{|c|c|c|c|c|}
\hline \multirow{2}{*}{ Warehouse } & \multicolumn{4}{|c|}{ Dealers } \\
\cline { 2 - 5 } & $\mathrm{D}_{1}$ & $\mathrm{D}_{2}$ & $\mathrm{D}_{3}$ & $\mathrm{D}_{4}$ \\
\hline $\mathrm{W}_{1}$ & .3749 & .4062 & .5287 & .5305 \\
\hline $\mathrm{W}_{2}$ & .3869 & .5322 & .5335 & .5194 \\
\hline $\mathrm{W}_{3}$ & .5284 & .5318 & .5246 & .5299 \\
\hline
\end{tabular}

Table 7: Most efficient index $\left(\boldsymbol{e}_{i j}^{\text {*** }}\right)$

\begin{tabular}{|c|c|c|c|c|}
\hline \multirow{2}{*}{ Warehouse } & \multicolumn{4}{|c|}{ Dealers } \\
\cline { 2 - 5 } & $\mathrm{D}_{1}$ & $\mathrm{D}_{2}$ & $\mathrm{D}_{3}$ & $\mathrm{D}_{4}$ \\
\hline $\mathrm{W}_{1}$ & .7089 & .7659 & 1 & 1 \\
\hline $\mathrm{W}_{2}$ & .7305 & 1 & 1 & 1 \\
\hline $\mathrm{W}_{3}$ & 1 & 1 & 1 & 1 \\
\hline
\end{tabular}

The optimal shipment plan to the above extended transportation problem(Table 2) is obtained by taking all three cases as discussed in section 5 one by one in following sub-sections.

7.2Optimal solution to ESTP

\subsubsection{Assume only availabilities are random variables}

Let us suppose that availabilities $A_{i} ; i=1,2,3$ follows normal distribution with known means, variances and specified probability levels as given in Table 8 .

Table 8: Means, variances and specified probability levels for warehouses

\begin{tabular}{|c|c|c|c|}
\hline Warehouse & Mean & Variance & Specified probability level \\
\hline $\mathrm{W}_{1}$ & $\mu_{1}=45$ & $\sigma_{1}^{2}=1$ & $\alpha_{1}=0.02$ \\
\hline $\mathrm{W}_{2}$ & $\mu_{2}=25$ & $\sigma_{2}^{2}=4$ & $\alpha_{2}=0.03$ \\
\hline $\mathrm{W}_{3}$ & $\mu_{3}=30$ & $\sigma_{3}^{2}=9$ & $\alpha_{3}=0.04$ \\
\hline
\end{tabular}


Further, it is assumed that the demands are known to DM and given in Table 9.

Table 9: Demands per destination

\begin{tabular}{|c|c|c|c|c|}
\hline Destinations & $\mathrm{D}_{1}$ & $\mathrm{D}_{2}$ & $\mathrm{D}_{3}$ & $\mathrm{D}_{4}$ \\
\hline Demand & 20 & 25 & 40 & 15 \\
\hline
\end{tabular}

By using information given in Table 8 and Table 9, we write model 3 as below.

Minimize $\sum_{i=1}^{3} \sum_{j=1}^{4}\left(1-e_{i j}\right) t_{i j}$

Subject to:

$$
\begin{aligned}
\sum_{j=1}^{4} t_{1 j} & \leq 42.9463 \\
\sum_{j=1}^{4} t_{2 j} & \leq 21.2384 \\
\sum_{j=1}^{4} t_{3 j} & \leq 24.7479 \\
\sum_{i=1}^{3} t_{i 1} & \geq 20 \\
\sum_{i=1}^{3} t_{i 2} & \geq 25 \\
\sum_{i=1}^{3} t_{i 3} & \geq 40 \\
\sum_{i=1}^{3} t_{i 4} & \geq 15 \quad, i=1,2,3 ; j=1,2,3,4 . \\
t_{i j} & \geq 0 \quad
\end{aligned}
$$

The above problem is an unbalanced transportation problem and it is converted to balance by adding dummy warehouse $\left(\mathrm{W}_{4}\right)$ with availability equals to excess demand. The optimal solution to balanced Model 3 is obtained by taking composite efficiency index $\boldsymbol{e}_{i j}^{*}$ in place of $\boldsymbol{e}_{i j}$ of equation (48). Hence, we have respective optimal solution to the above problem as below.

$t_{12}=25, t_{13}=18, t_{23}=21, t_{31}=10, t_{34}=15, t_{41}=10, t_{43}=1$, with the optimum value of objective function as 41.2356 .

Similarly, the optimal solution to balanced Model 3 is obtained by replacing $\boldsymbol{e}_{i j}$ in equation (48) by composite efficiency index $\boldsymbol{e}_{i j}^{* *}$. Hence, we have respective optimal solution as below.

$t_{13}=40, t_{14}=3, t_{22}=21, t_{31}=20, t_{32}=4, t_{34}=1, t_{44}=11$, with the optimum value of objective function as zero.

\subsubsection{Assume only demands are random variables}

Let us suppose that demands $\boldsymbol{B}_{j} ; j=1,2,3,4$. follow normal distribution with known means, variances and specified probability levels as given in Table 10.

Table 10: Means, variances and specified probability levels for destinations

\begin{tabular}{|c|c|c|c|}
\hline Destinations & Mean & Variance & Specified probability level \\
\hline $\mathrm{D}_{1}$ & $\mu_{1^{\prime}}^{\prime}=20$ & $\sigma_{1}^{\prime 2}=1$ & $\beta_{1}=0.03$ \\
\hline $\mathrm{D}_{2}$ & $\mu_{2}^{\prime}=35$ & $\sigma_{2}^{\prime 2}=4$ & $\beta_{2}=0.04$ \\
\hline $\mathrm{D}_{3}$ & $\mu_{3}^{\prime}=25$ & $\sigma_{3}^{\prime 2}=9$ & $\beta_{3}=0.05$ \\
\hline $\mathrm{D}_{4}$ & $\mu_{4}^{\prime}=15$ & $\sigma_{4}^{\prime 2}=4$ & $\beta_{4}=0.06$ \\
\hline
\end{tabular}


Further, it is assumed that the availabilities are known to DM and given in Table 11.

Table 11: Availabilities per warehouse

\begin{tabular}{|c|c|c|c|}
\hline Warehouse & $\mathrm{W}_{1}$ & $\mathrm{~W}_{2}$ & $\mathrm{~W}_{3}$ \\
\hline Availability & 20 & 45 & 30 \\
\hline
\end{tabular}

By using information given in Table 10 and Table 11, we write model 4 as below.

Minimize $\sum_{i=1}^{3} \sum_{j=1}^{4}\left(1-e_{i j}\right) t_{i j}$

Subject to:

$$
\begin{aligned}
& \sum_{j=1}^{4} \boldsymbol{t}_{1 j} \leq 20 \\
& \sum_{j=1}^{4} \boldsymbol{t}_{2 j} \leq 45 \\
& \sum_{j=1}^{4} \boldsymbol{t}_{3 j} \leq 30 \\
& \sum_{i=1}^{3} \boldsymbol{t}_{i 1} \geq 21.8807 \\
& \sum_{i=1}^{3} \boldsymbol{t}_{i 2} \geq 35.5013 \\
& \sum_{i=1}^{3} \boldsymbol{t}_{i 3} \geq 29.9345 \\
& \sum_{i=1}^{3} \boldsymbol{t}_{i 4} \geq 18.5013 \\
& \boldsymbol{t}_{i j} \geq 0 \quad, i=1,2,3 ; j=1,2,3,4 .
\end{aligned}
$$

The above problem is an unbalanced transportation problem and it is converted to balance by adding dummy warehouse $\left(\mathrm{W}_{4}\right)$ with availability equals to excess demand. The optimal solution to balanced Model 4 is obtained by taking composite efficiency index $\boldsymbol{e}_{i j}^{*}$ in place of $\boldsymbol{e}_{i j}$ of equation (49). Hence, we have respective optimal solution to the above problem as below.

$t_{12}=20, t_{22}=15, t_{23}=30, t_{31}=10, t_{32}=1, t_{34}=19, t_{41}=12$, with the optimum value of objective function as 43.2522 .

Similarly, the optimal solution to balanced Model 4 is obtained by replacing $\boldsymbol{e}_{i j}$ in equation (49) by composite efficiency index $\boldsymbol{e}_{i j}^{* *}$. Hence, we have respective optimal solution as below.

$t_{13}=20, t_{22}=36, t_{23}=9, t_{31}=22, t_{33}=1, t_{34}=7, t_{44}=12$, with the optimum value of objective function as zero.

\subsubsection{Both availability and demand follows normal distribution}

Suppose availability at $\mathrm{i}^{\text {th }}$ warehouse $\left(A_{i}, i=1,2,3\right.$ ) follows normal distribution with known means, variances and specified probability levels as given in Table 12 .

Suppose demand at $\mathrm{j}^{\text {th }}$ destination ( $\boldsymbol{B}_{j}, j=1,2,3,4$. ) follows normal distribution with known means, variances and specified probability levels as given in Table 13. 
Table 12: Means, variances and specified probability levels for warehouses

\begin{tabular}{|c|c|c|c|}
\hline Warehouse & Mean & Variance & Specified probability level \\
\hline $\mathrm{W}_{1}$ & $\mu_{1}=25$ & $\sigma_{1}^{2}=1$ & $\alpha_{1}=0.02$ \\
\hline $\mathrm{W}_{2}$ & $\mu_{2}=15$ & $\sigma_{2}^{2}=4$ & $\alpha_{2}=0.03$ \\
\hline $\mathrm{W}_{3}$ & $\mu_{3}=20$ & $\sigma_{3}^{2}=4$ & $\alpha_{3}=0.04$ \\
\hline
\end{tabular}

Table 13: Means, variances and specified probability levels for destinations

\begin{tabular}{|c|c|c|c|}
\hline Destinations & Mean & Variance & Specified probability level \\
\hline $\mathrm{D}_{1}$ & $\mu_{1^{\prime}}^{\prime}=10$ & $\sigma_{1}^{\prime 2}=1$ & $\beta_{1}=0.03$ \\
\hline $\mathrm{D}_{2}$ & $\mu_{2}^{\prime}=12$ & $\sigma_{2}^{\prime 2}=4$ & $\beta_{2}=0.04$ \\
\hline $\mathrm{D}_{3}$ & $\mu_{3}^{\prime}=15$ & $\sigma_{3}^{\prime 2}=9$ & $\beta_{3}=0.05$ \\
\hline $\mathrm{D}_{4}$ & $\mu_{4}^{\prime}=5$ & $\sigma_{4}^{\prime 2}=4$ & $\beta_{4}=0.06$ \\
\hline
\end{tabular}

By using information given in Table 12 and Table 13, we write model 5 as below.

$$
\text { Minimize } \sum_{i=1}^{3} \sum_{j=1}^{4}\left(1-e_{i j}\right) t_{i j}
$$

Subject to:

$$
\begin{aligned}
& \sum_{j=1}^{4} t_{1 j} \leq 22.94 \\
& \sum_{j=1}^{4} t_{2 j} \leq 11.24 \\
& \sum_{j=1}^{4} t_{3 j} \leq 16.5 \\
& \sum_{i=1}^{3} t_{i 1} \geq 11.88 \\
& \sum_{i=1}^{3} t_{i 2} \geq 15.50 \\
& \sum_{i=1}^{3} t_{i 3} \geq 19.92 \\
& \sum_{i=1}^{3} t_{i 4} \geq 8.12 \\
& t_{i j} \geq 0 \quad, i=1,2,3 ; j=1,2,3,4 .
\end{aligned}
$$

The above problem is an unbalanced transportation problem and it is converted to balance by adding dummy warehouse $\left(\mathrm{W}_{4}\right)$ with availability equals to excess demand. The optimal solution to balanced Model 5 is obtained by taking composite efficiency index $\boldsymbol{e}_{i j}^{*}$ in place of $\boldsymbol{e}_{i j}$ in equation (50). Hence, we have respective optimal solution to the above problem as below.

$t_{12}=16, t_{13}=7, t_{23}=11, t_{31}=9, t_{34}=8, t_{41}=3, t_{43}=2$, with the optimum value of objective function as 23.3368 . 
Similarly, the optimal solution to balanced Model 5 is obtained by replacing $\boldsymbol{e}_{i j}$ in equation (50) by composite efficiency index $\boldsymbol{e}_{i j}^{* *}$. Hence, we have respective optimal solution as below.

$t_{13}=20, t_{14}=3, t_{22}=11, t_{31}=12, t_{32}=5, t_{42}=0, t_{44}=5$, with the optimum value of objective function as zero.

\section{Conclusion}

We have discussed the method to solve extended stochastic transportation problem with multiple flexible inputs and multiple outputs per shipment link (DMUs). The relative efficiency concept is used to decide the optimal shipment per DMUs. DEA technique, input oriented BCC model, is used to calculate efficiencies for DMUs. Two kinds of relative efficiency are incorporated into the solution procedure using different set of DMUs. A composite efficiency index is defined to measure the performance of a particular shipment link. The optimal shipment plan is decided by taking into account the most efficient DMUs. The suggested approach is more applicable to real life transportation problem under the uncertainty of the availability and demand. It is assumed that either availabilities or demands or both are random variables and follows normal distribution with known parameters.

\section{References}

[1]. Abdul Quddoos, Md GulzarHasan and Mohammad Masood Khalid.(2014) 'Multi-choice stochastic transportation problem involving general form of distributions' Springer Plus, Vol.No.3:565.

[2]. Alireza Amirteimoori(2012) 'An extended transportation problem: a DEA-based approach' Central European Journal of Operations research,Vol.19(5):513-521.

[3]. Banker R.D.,Charnes A. and Cooper W.W.(1984) 'Some methods for estimating technical and scale inefficiencies in data envelopment analysis' Management Science,Vol.No.30(9): 1078-1092.

[4]. Biswal M.P.and Samat H.K.(2013) 'Stochastic transportation problem with Cauchy random variables and multi-choice parameters' Journal of physical sciences, Vol.No.17:117-130.

[5]. Charnes A. and Cooper W.W.(1962) 'Programming with linear fractional functions' Naval research logistic quarterly, Vol.No.9:181-186.

[6]. Charnes A., Cooper W.W., and Rhodes E.(1978) 'Measuring the efficiency of decision making units' European journal of operational research' Vol.No.2(6):429-444.

[7]. Chen L.H. and Lu H.W.(2007) 'An extended assignment problem considering multiple inputs and outputs' Applied mathematical modeling,Vol.No.31:2239-2248.

[8]. Gedam V.Kand Pathan M.B.(2014) 'An extended transportation problem with multiple inputs and outputs' IOSR Journal of Mathematics,Vol.10(6):Ver.V:58-63.

[9]. Gedam V.K. and Pathan M.B.(2016) 'Two stage approach to solve extended transportation problem' International Journal of Mathematics and Soft Computing, Vol.6(2):111-119.

[10]. Gedam V.K and Pathan M.B(2016) 'Common set of weights approach to solve extended transportation problem with chance constraints' Asian Journal of Mathematics and Computer Research, Vol.13(4):245-255.

[11]. Gedam V.K and Pathan M.B(2016) 'Two stage approach to solve extended transportation problem with probability constraints' Asian Journal of Mathematics and Computer Research, Vol.14(4):333-342.

[12]. Hitchcock F.L.(1941) 'The distribution of a product from several sources to numerous localities' Journal of mathematics and physics, Vol.No.20:224-230.

[13]. Maurya V.N.,Misra R.B.,Jaggi C.K. and Maurya A.K.(2014) 'Progressive review and analytical approach for optimal solution of stochastic transportation problems involving multi-choice cost'American journal of modeling and optimization, Vol.No.2:77-83.

[14]. Roy S.K.,Mahapatra D. R.and Biswal M.P.(2010) 'Multi-objective stochastic transportation problem involving log-normal' Journal of physical sciences,Vol.No.14:63-76.

[15]. Roy S.K. and Mahapatra D. R.(2011) 'Multi-objective interval-valued transportation probabilistic problem involving log-normal' International journal of mathematics and scientific computing,Vol.No.1(2):14-21.

[16]. Roy S.K.,Mahapatra D. R. and Biswal M.P.(2012) 'Multi-objective stochastic transportation problem with exponential distribution' Journal of uncertain systems, Vol.No.6(3):200-213. 